\title{
The Adventitia May Be a Barrier Specific to Nitric Oxide in Rabbit Pulmonary Artery
}

\author{
Robin H. Steinhorn, Frederick C. Morin III, and James A. Russell \\ Departments of Physiology and Pediatrics, State University of New York at Buffalo, Buffalo, New York 14214
}

\begin{abstract}
To determine whether the adventitia that surrounds pulmonary vessels acts as a barrier specific to nitric oxide, special lucite chambers were constructed to measure the force of contraction of rabbit pulmonary artery rings in which the endothelial or adventitial surfaces could be preferentially exposed to nitric oxide (NO), carbon monoxide (CO), or sodium nitroprusside (SNP). Delivery of NO to the endothelial and adventitial surfaces of preconstricted vessels produced markedly different concentration-response curves with maximal relaxations of $89 \pm 3$ and $11 \pm 9 \%$, respectively. In contrast, relaxations induced by both $C O$ and SNP did not differ significantly between endothelial and adventitial exposure to these agents. Placement of a layer of pericardium onto the endothelial surface eliminated relaxation to the endothelial delivery of $\mathrm{NO}$ but not to $\mathrm{CO}$. We conclude that the pulmonary vascular response to NO displays a striking sidedness which is not observed either with $\mathrm{CO}$, another gas of similar molecular weight, or with SNP, both of which cause relaxation by stimulating guanylate cyclase. The elimination of $\mathrm{NO}$ but not $\mathrm{CO}$ relaxations with a layer of pericardium may indicate that the adventitia acts as a barrier specific to NO. This directionality of effect provides evidence for a highly localized regulation of pulmonary vascular tone by endothelial cell NO and also indicates that extravascular NO may have limited access to pulmonary vascular smooth muscle. (J. Clin. Invest. 1994. 94:18831888.) Key words: nitric oxide • endothelium • in vitro studies • vascular smooth muscle • lung
\end{abstract}

\section{Introduction}

The mechanism by which nitric oxide (NO) regulates vascular tone has been studied extensively over the past decade (1-3). It is now clear that NO can be produced by several forms of NO synthase that are present in many different cell types (4, $5)$. Thus, in addition to endothelial cells, NO may be produced by macrophages (6), mast cells (7), neutrophils (8), and even vascular smooth muscle cells (9). Moreover, intracellular regulatory mechanisms for NO, and the amounts of NO produced, vary widely among cell types, depending on constitutive versus inducible forms of NO synthase as well as whether the NO

Address correspondence to Robin H. Steinhorn, M.D., Children's Hospital at Buffalo, 219 Bryant Street, Buffalo, NY 14222.

Received for publication 16 February 1994 and in revised form 2 June 1994.

J. Clin. Invest.

(C) The American Society for Clinical Investigation, Inc.

$0021-9738 / 94 / 11 / 1883 / 06 \$ 2.00$

Volume 94, November 1994, 1883-1888 produced is destined for the regulation of vascular smooth muscle tone, platelet aggregation, or cytotoxic effects $(4,5)$.

Pulmonary vascular tone is regulated by the smooth muscle layer that encircles both pulmonary arteries and veins. This layer of smooth muscle is in the unique position of potentially being influenced by NO produced by both endothelial cells and extravascular sources such as activated macrophages, mast cells, or neutrophils that are recruited into the lung during periods of inflammation. If the NO produced by these extravascular sources gains ready access to the pulmonary vascular smooth muscle across the adventitia, then it might interfere with normal regulation of vascular tone by endothelial cells and produce a general state of pulmonary vasodilation.

Exogenous NO gas delivered via the airspaces is a selective pulmonary vasodilator in awake lambs (10-12). These findings have led to the development of clinical studies using inhaled NO to treat various forms of pulmonary hypertension. It has been presumed, but not determined, that this inhaled NO gains access to the pulmonary vascular smooth muscle by traversing the adventitia (10).

The purpose of this study was to investigate whether the adventitial layer that surrounds pulmonary vessels might act as a barrier to prevent extravascular sources of NO from influencing pulmonary vascular tone. To test this hypothesis, a new tissue holder was developed. This device has the advantage of directly measuring the force of contraction of isolated rabbit pulmonary arteries while the endothelial and adventitial surfaces are superperfused separately. Exogenous NO gas delivered to the endothelial surface produced markedly greater relaxations than NO delivered to the adventitial surface.

\section{Methods}

This study was approved by the State University of New York at Buffalo Laboratory Animal Care Committee. Rabbits weighing between 2.5 and $4.0 \mathrm{~kg}$ were anesthetized with intravenous pentobarbital sodium (30 $\mathrm{mg} / \mathrm{kg}$ ) and killed by exsanguination. The trachea, lungs, and heart were removed from the thorax immediately after death and placed in Krebs-Ringer solution ( $118 \mathrm{mM} \mathrm{NaCl}, 4.7 \mathrm{mM} \mathrm{KCl}, 2.5 \mathrm{mM} \mathrm{CaCl}{ }_{2}$, $1.2 \mathrm{mM} \mathrm{MgSO}_{4}, 1.2 \mathrm{mM} \mathrm{KH}_{2} \mathrm{PO}_{4}, 25.5 \mathrm{mM} \mathrm{NaHCO}_{8}, 5.6 \mathrm{mM}$ glucose, and $0.026 \mathrm{mM}$ EDTA). Portions of intrapulmonary and extrapulmonary arteries with inside diameters of 2-3 mm were dissected into rings $\sim 1$ $\mathrm{cm}$ long. Each ring was then cut longitudinally and flattened out. In selected tissues, the endothelium was removed by gently rubbing the intimal surface with curved forceps for $\sim 45 \mathrm{~s}$ before cutting longitudinally. Adequate removal of the endothelium was confirmed by the absence of any relaxation to $10^{-8}-10^{-6} \mathrm{M}$ acetylcholine applied to the endothelial surface of pulmonary arteries contracted by an $\mathrm{ED}_{50}$ of phenylephrine.

Each cut pulmonary artery segment was placed in a specially designed and constructed lucite tissue holder (Fig. 1) that permits separate perfusion of the endothelial and adventitial surfaces while allowing direct measurement of the force of contraction. The tissue holder consists of upper and lower chambers that can be clamped together with the vessel sandwiched between, thus exposing round $0.125-\mathrm{cm}^{2}$ areas $(4$ 


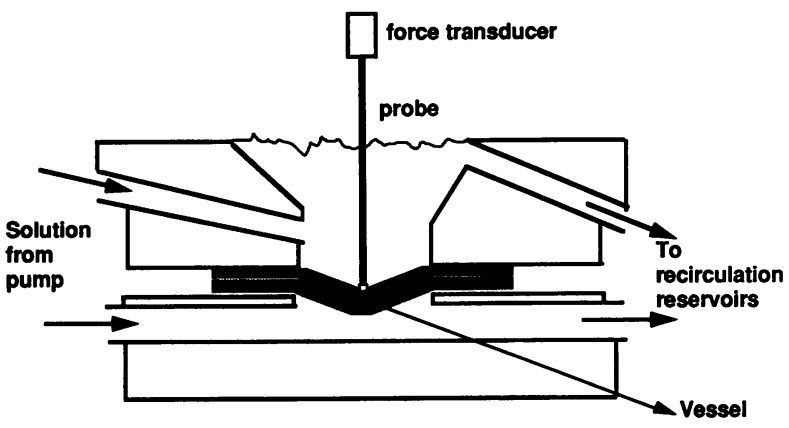

Figure 1. Diagram of the tissue holder used separately to perfuse the endothelial or adventitial surfaces of rabbit pulmonary arteries. Perfusion media entered the upper and lower chambers from the left side via a reservoir and heat exchanger in line with a roller pump. After passing over the upper or lower surfaces of the tissue, perfusion media was returned to the reservoir for recirculation. A probe connected to a force transducer was used to depress the tissue and detect changes in the force of contraction.

mm diameter) on opposite sides of the tissue. The upper and lower surfaces of the tissue were independently perfused at a rate of $5 \mathrm{ml} /$ min with Krebs-Ringer solution using a variable speed peristaltic pump (Minipuls 3; Gilson Medical Electronics, Inc., Middleton, WI) installed between the tissue holder and two glass reservoirs (one each for the upper and lower chambers). Total recirculating volume in each chamber-reservoir system was $6 \mathrm{ml}$. An aerator in each reservoir was used to equilibrate the Krebs-Ringer solution with a gas mixture of $94 \% \mathrm{O}_{2}$ / $6 \% \mathrm{CO}_{2}$ to obtain a pH of 7.4, a $\mathrm{CO}_{2}$ tension of 38 torr and an $\mathrm{O}_{2}$ tension of $>500$ torr. Each reservoir allowed free access for the addition of drugs or gases and fresh Krebs-Ringer solution as needed. The KrebsRinger solution was maintained at $37^{\circ} \mathrm{C}$ by a heated water jacket around each reservoir, as well as a heat exchanger placed in line just before the inlets to the upper and lower chambers. Four complete tissue holders were available permitting four pulmonary artery segments from each rabbit to be investigated simultaneously.

A continuous recording of isometric force was obtained by depressing a stainless steel probe tipped with polyvinylchloride onto the exposed upper surface of the tissue. The probe was connected to a force transducer (Statham UC 2; Statham Instruments, Hato Rey, PR) connected to an oscillographic recorder (four channel chart recorder \#7414A; Hewlett-Packard Co., Palo Alto, CA). No pulsatile changes in the isometric force recording due to the peristaltic pump were noted. Preliminary experiments revealed that a passive tension of $800 \mathrm{mg}$ is required for maximum active force generation to $10^{-6} \mathrm{M}$ phenylephrine. This passive tension was achieved by depressing each tissue $\sim 1.5 \mathrm{~mm}$ with the probe. After the optimal passive tension was obtained, the tissues were allowed to equilibrate in recirculating $37^{\circ} \mathrm{C}$ Krebs-Ringer solution for $45 \mathrm{~min}$ before instituting experimental protocols.

Because the Krebs-Ringer solution in the upper chamber was exposed to air, gases such as $\mathrm{NO}$ and $\mathrm{CO}$ were delivered only to the lower chamber in order to minimize gas loss. Therefore, comparisons were made between pulmonary artery segments mounted either endothelial side down or adventitial side down for all experimental protocols except those described in Fig. 3. NO and $\mathrm{CO}$ were delivered in a cumulative manner by bubbling $1 \mathrm{cc}$ of each gas concentration into the KrebsRinger solution of the lower chamber reservoir over a 15-s period. The response to each concentration of $\mathrm{NO}$ or $\mathrm{CO}$ was allowed to plateau before the next higher concentration was added. Serial dilutions of NO in nitrogen were made from a cylinder containing $965 \mathrm{ppm}$ NO in nitrogen, and were injected into the reservoirs in concentrations ranging from 1 to $965 \mathrm{ppm}$. Serial dilutions of $\mathrm{CO}$ in nitrogen were made from a cylinder of $100 \% \mathrm{CO}$, and were injected into the reservoirs in concentrations ranging from 0.1 to $100 \%$. These values are used in the generation of subsequent concentration-response curves for NO and

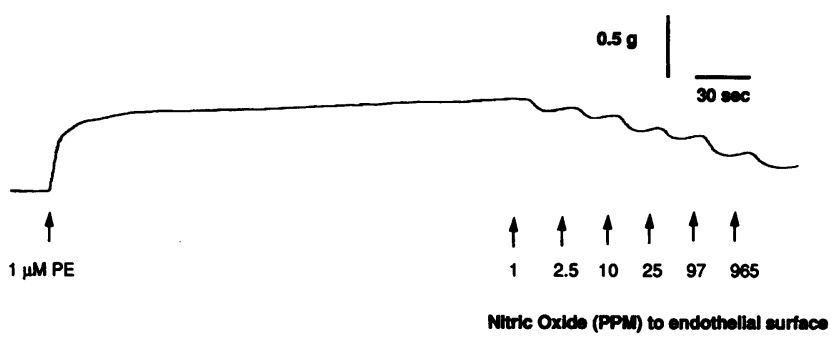

Figure 2. Original record showing the contractile response to $1 \mu \mathrm{M}$ phenylephrine (PE) applied to both the endothelial and adventitial surfaces, followed by cumulative additions of NO to the solution perfusing the endothelial (lower) surface only.

$\mathrm{CO}$ (see Figs. 2-7). The actual concentrations of $\mathrm{NO}$ and $\mathrm{CO}$ reaching the tissues were not known, but were substantially less than the injected concentrations, as the reservoirs were open to air. Due to the rapid inactivation of NO by superoxide anion, vessels were incubated on both sides with $150 \mathrm{U} / \mathrm{ml}$ of SOD throughout all experimental protocols. Protocols involving the use of sodium nitroprusside (SNP) ${ }^{1}$ were performed in a darkened room in order to prevent the photochemical release of NO (13).

Drugs used in this study included acetylcholine chloride, phenylephrine hydrochloride, SNP, and SOD. All drugs were purchased from Sigma Chemical Co. (St. Louis, MO). All drugs were dissolved in deionized water just before experimentation. Drugs were added to the 6-ml perfusion solution in aliquots of $\langle 50 \mu \mathrm{l}$ and were removed by repeated washing over a $45-$ min period when applicable. NO gas was obtained from Matheson Gas Products (Twinsburg, $\mathrm{OH}$ ) and $\mathrm{CO}$ gas was obtained from Linde (Danbury, CT).

In all experiments, $n$ represents the number of animals from which vessel rings were studied. Data are expressed as means \pm SEM. Statistical comparison was performed on concentration-response curves using ANOVA with Student-Newman-Keuls test for post hoc testing of multiple comparisons, when appropriate. Individual data points between curves were compared using Students' unpaired $t$ test. Significance was accepted at $P<0.05$.

\section{Results}

A typical protocol is shown in Fig. 2. This pulmonary artery segment was mounted endothelial surface down and it contracted vigorously in response to an $\mathrm{ED}_{50}$ of phenylephrine delivered to both the endothelial and adventitial surfaces simultaneously. Once the contraction reached a plateau, NO was injected into the reservoir supplying the endothelial (lower) surface in increasing concentrations as shown. NO produced a marked concentration-dependent relaxation of this pulmonary artery.

To demonstrate that chamber design did not influence the results, eight vessel segments were studied, using each vessel as its own control. One group of four vessels was mounted endothelial surface up, and the second group of four was mounted endothelial surface down. In half of the vessels in each group, NO was delivered first to the upper chamber and then to the lower chamber after a wash in between and subsequent readdition of phenylephrine. The other two vessels in each group received the reverse order of NO delivery. Mean was

1. Abbreviations used in this paper: EDRF, endothelium-derived relaxing factor; SNP, sodium nitroprusside. 

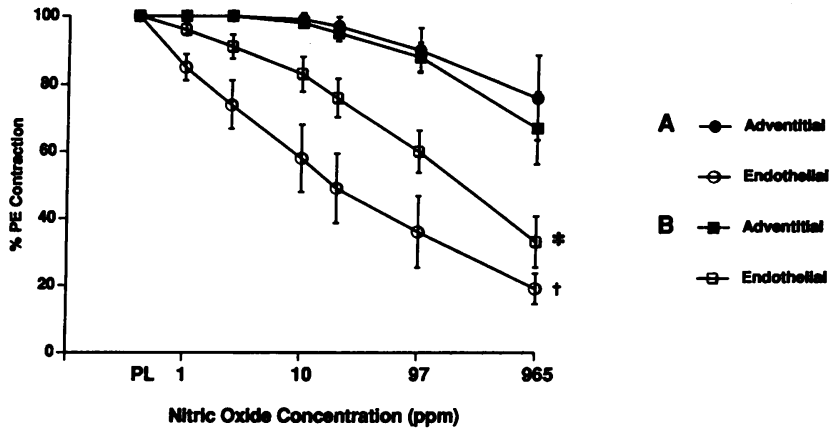

Figure 3. Comparison of NO concentration-response curves between endothelial and adventitial exposures to NO. $(A)$ vessels mounted endothelial surface up. $(B)$ vessels mounted adventitial surface up. Relaxations are expressed as percent plateau PE contraction. *, ${ }^{\ddagger}$ significantly different from adventitial NO in groups A and B, respectively. $n=4$ for each curve. PL, plateau contraction (100\%) to $1 \mu \mathrm{M}$ phenylephrine (Plateau contraction force: group $\mathrm{A}=698 \pm 92 \mathrm{~g}$ for endothelial NO and $676 \pm 102 \mathrm{~g}$ for adventitial NO. Group B $=669 \pm 98 \mathrm{~g}$ for endothelial NO and $725 \pm 109 \mathrm{~g}$ for adventitial NO.)

calculated with all data taken together and the results are shown in Fig. 3. Regardless of which vessel orientation was examined, the endothelial delivery of NO always produced a substantially greater degree of relaxation than the adventitial delivery of NO. However, the endothelial delivery of NO produced the least relaxation when the vessels were mounted endothelial surface up, because the solution in the upper chamber was exposed to air, allowing some NO to escape. For this reason, all subsequent protocols were performed with delivery of NO, CO, or SNP to the lower chamber only.

A comparison of endothelial versus adventitial exposure to $\mathrm{NO}$ is shown in Fig. 4. In contrast to the dramatic relaxation observed when NO was delivered to the endothelial surface, very little effect was observed when NO was delivered to the

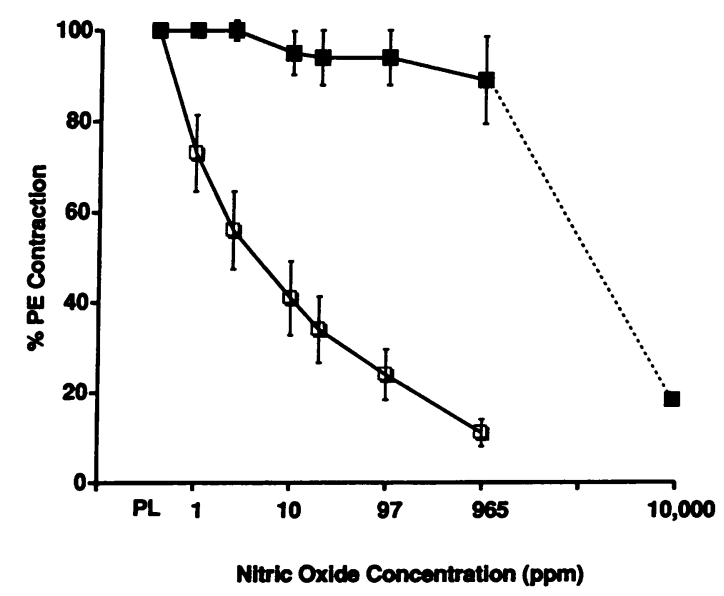

Figure 4. Comparison of NO concentration-response curves between endothelial ( $\square$ ) and adventitial ( $\square$ ) exposures to this agent. Relaxations are expressed as percent plateau PE contraction. The two curves were significantly different $(P<0.01)$ at each concentration of NO. $n=6$ for each curve. Broken line represents relaxation following adventitial exposure to $10 \%$ NO $(n=2)$. PL, plateau contraction $(100 \%)$ to 1 $\mu \mathrm{M}$ phenylephrine. (Plateau contraction force $=675 \pm 97 \mathrm{~g}$ for endothelial exposure and $722 \pm 76 \mathrm{~g}$ for adventitial exposure.)

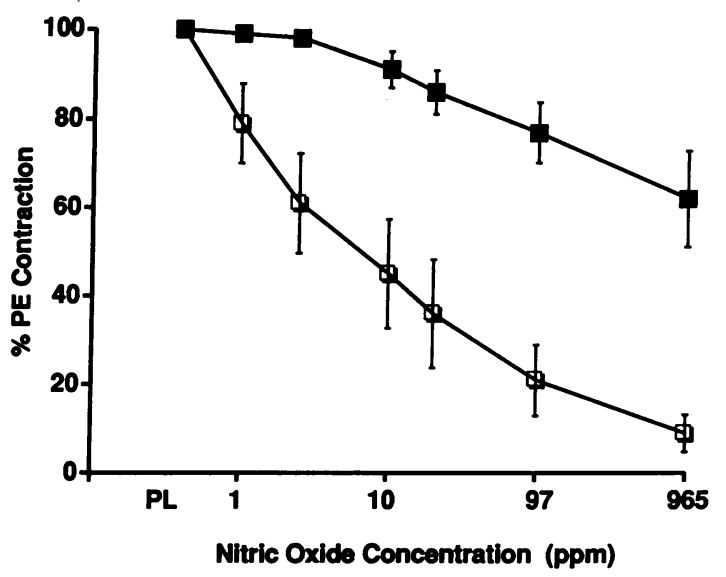

Figure 5. Comparison of NO concentration-response curves between endothelial ( $\square$ ) and adventitial ( $\square$ ) exposures in endothelium-denuded pulmonary arteries. Relaxations are expressed as percent plateau PE contraction. The two curves were significantly different $(P<0.01)$ at each concentration of NO. $n=6$ for each curve. PL, plateau contraction $(100 \%)$ to $1 \mu \mathrm{M}$ phenylephrine. (Plateau contraction force $=625 \pm 54$ $\mathrm{g}$ for endothelial exposure and $675 \pm 65 \mathrm{~g}$ for adventitial exposure.)

adventitial surface of pulmonary artery segments. Relaxations to NO could be abolished by first incubating vessels with 10 $\mu \mathrm{M}$ hemoglobin, a scavenger of NO (data not shown, $n=2$ for both endothelial and adventitial exposure). In three of the six vessels exposed to adventitial NO, the highest concentration of NO (965 ppm) was delivered two more times at 1-min intervals after the initial exposure to $965 \mathrm{ppm}$ NO. The level of relaxation was not enhanced after these repetitive exposures to $965 \mathrm{ppm}$ NO. However, adventitial exposure to $10 \%$ NO $(100,000 \mathrm{ppm}$ ) relaxed vessels by $86 \%$ (Fig. $4, n=2)$. The difference between endothelial and adventitial delivery of NO persisted in endothelium-denuded vessels (Fig. 5), although the relaxation response to adventitial NO was somewhat greater than in endothelium-intact vessels (Fig. 4) at NO concentrations $>10$ ppm.

A layer of pericardium was dissected from around the rabbit heart and placed in contact with the endothelial surface in another group of pulmonary arteries. This layer of pericardium significantly reduced the level of relaxation to endothelial delivery of NO when compared with control (Fig. 6). Indeed, this NO concentration-response curve in the presence of the pericardium does not differ significantly from the adventitial delivery of NO (Fig. 4) at any concentration of NO. In this protocol, the pericardium was removed after the initial NO concentration-response curve was obtained and the tissue was remounted endothelial surface down. Removal of the pericardium restored responses to the endothelial delivery of NO (data not shown).

$\mathrm{CO}$ also relaxed pulmonary arteries contracted by an $\mathrm{ED}_{50}$ of phenylephrine, although to a lesser degree than NO (Fig. 7). In marked contrast to the results obtained with NO, endothelial versus adventitial $\mathrm{CO}$ concentration-response curves were not significantly different $(P>0.05)$ at any $\mathrm{CO}$ concentration. Moreover, addition of a layer of pericardium to the endothelial surface had no effect on relaxations to $\mathrm{CO}$.

There was no significant difference between the relaxations elicited by SNP delivered to either the endothelial or adventitial surfaces of rabbit pulmonary arteries (Fig. 8). 


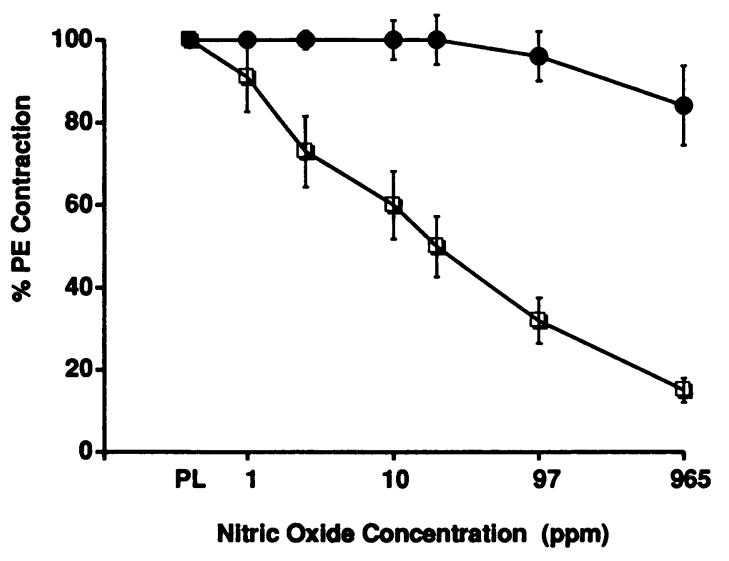

Figure 6. Comparison of NO concentration-response curves in two groups of vessels mounted endothelial surface down. In one group a layer of rabbit pericardium was placed over the endothelial surface $(\bullet)$. Relaxations are expressed as percent plateau PE contraction. The two curves were significantly different $(P<0.01)$ at NO concentrations $>1 \mathrm{ppm} . n=6$ for each curve. PL, plateau contraction $(100 \%)$ to 1 $\mu \mathrm{M}$ phenylephrine. (Plateau contraction force $=652 \pm 66 \mathrm{~g}$ for endothelial exposure and $672 \pm 59 \mathrm{~g}$ for endothelial+pericardium exposure.)

\section{Discussion}

Delivery of NO to the endothelial surface of isolated rabbit pulmonary arteries contracted by phenylephrine produced significantly greater relaxation than delivery of NO to the adventitial surface. To ensure that this differential effect of NO was not caused by some factor related to the design of this new tissue holder, the results of three different protocols were compared. Regardless of whether a tissue was mounted $(a)$ endothelial or adventitial surface up, $(b)$ used as its own control, or (c) compared with an adjacent tissue, the endothelial delivery of NO always elicited the greatest relaxation.

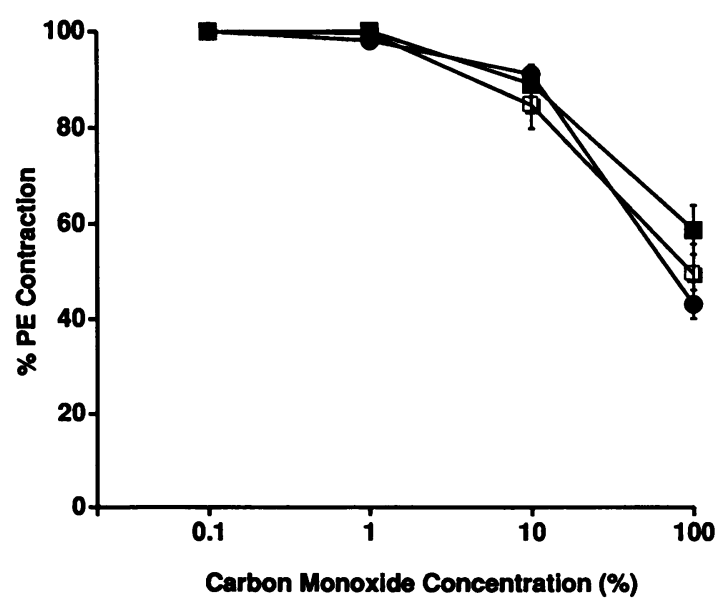

Figure 7. Comparison of $\mathrm{CO}$ concentration-response curves among endothelial exposure ( $\square$ ), adventitial exposure ( $\square$ ), and endothelial+pericardium exposure $(\bullet)$. Relaxations are expressed as percent plateau contraction to $1 \mu \mathrm{M}$ phenylephrine. The three curves were not significantly different at any concentration of $\mathrm{CO}$. $n=6$ for each curve. (Plateau contraction force $=688 \pm 67 \mathrm{~g}$ for endothelial exposure, $701 \pm 66 \mathrm{~g}$ for adventitial exposure, and $696 \pm 56 \mathrm{~g}$ for endothelial \pm pericardium exposure.)

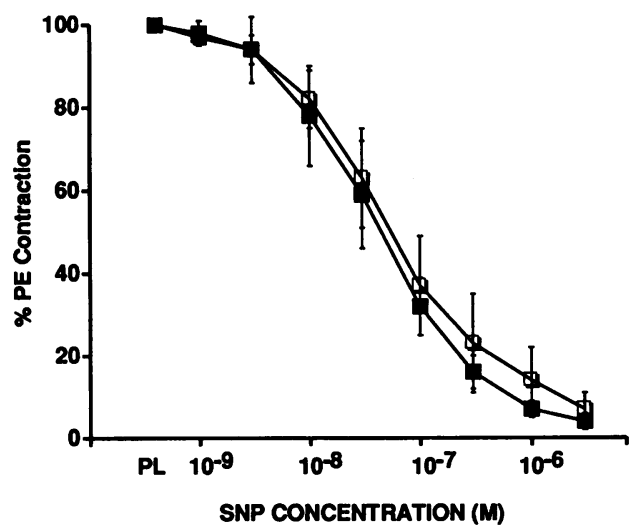

Figure 8. Comparison of SNP concentration-response curves between endothelial ( $\square$ ) and adventitial ( $\square$ ) exposures to this agent. The two curves were not significantly different at any concentration of SNP. $n$ $=6$ for each curve. PL, plateau contraction ( $100 \%)$ to $1 \mu \mathrm{M}$ phenylephrine. (Plateau contraction force $=668 \pm 64 \mathrm{~g}$ for endothelial exposure and $675 \pm 58 \mathrm{~g}$ for adventitial exposure.)

The striking sidedness of the response to NO suggests that either the endothelial cell layer or the adventitia might regulate access of exogenous NO gas to the underlying layer of smooth muscle. Removal of the endothelium did not alter the differential response to NO, indicating that this cell layer is not involved. Attempts to remove the adventitia either by mechanical dissection or enzymatic digestion were not successful because small holes eventually developed in the remaining tissue. This likely disrupted contractile mechanisms and allowed NO to pass from one side of the tissue to the other.

An alternative to removal of the adventitia was to place an adventitia-like layer of elastin and collagen over the endothelial surface and test its ability to prevent access of NO to the smooth muscle layer. In this study, a layer of pericardium significantly reduced the level of relaxation to the endothelial delivery of NO. The pericardium was effective whether the endothelium was intact or denuded (data not shown) and removal of the pericardium restored responses to the endothelial delivery of NO. Thus, the adventitia may prevent exogenous NO gas from gaining access to the underlying layer of smooth muscle. This hypothesis receives support from the current study, as the effect of NO delivered to the adventitial surface was slightly greater in endothelium-denuded than in intact vessels. It is likely that the mechanical process used to remove the endothelium also produces some abrasion of the adventitia and compromises its ability to restrict NO access to the smooth muscle layer.

Two other studies using superfusion bioassay techniques with donor and recipient vessels have investigated the ability of endothelium-derived relaxing factor (EDRF) generated in response to acetylcholine to cross from the inside to the outside of the vessel wall. Vedernikov et al. (14) reported no transition for EDRF through the vessel wall of rabbit thoracic aorta and femoral artery, and concluded that local metabolic factors located in the adventitia can influence endothelial cell function. Bassenge et al. (15) reported similar findings in the same species and vessels and, in addition, found that EDRF from cultured bovine endothelial cells elicited smaller dilation $(9 \pm 4 \%)$ when applied to the adventitial side of endothelium-denuded vessels, compared with endothelial application $(92 \pm 7 \%)$. However, they reasoned that the adventitia was not a barrier because 
subsequent histological examination revealed that the adventitia was not a continuous layer in any vessel studied. A damaged adventitia probably resulted from trauma associated with the techniques used to remove the endothelium, as well as the eversion and inversion techniques they used to study their vessels. Instead, they attributed differences in endothelial versus adventitial exposure to EDRF to an outer layer of smooth muscle that was less sensitive to EDRF than the inner layer.

The differences between our study and those of Vedernikov et al. (14) and Bassenge et al. (15) are numerous. First, they studied major systemic blood vessels, while we studied smaller pulmonary blood vessels. In addition, they studied responses to EDRF generated by acetylcholine rather than exogenous NO gas and they did not use SOD in their superfusion systems. Thus, problems associated with small amounts of endogenously produced NO, the short half-life of NO due to rapid metabolism by superoxide anion, and dilution factors inherent to cascadetype superfusion systems were less of a concern with our unique tissue holder. Also, we used extreme care to avoid any damage to the adventitial surface and we also were able to demonstrate that an adventitial-like layer of pericardium placed over the endothelial surface could mimic the response to the adventitial delivery of NO.

In addition, we studied relaxations to SNP and $\mathrm{CO}$ to determine whether the differential response to NO was unique to this molecule. These agents were chosen because, like NO, they induce relaxation of vascular smooth muscle by stimulating guanylate cyclase. SNP is a potent vasodilator that causes smooth muscle relaxation by releasing NO, which then activates guanylate cyclase $(13,16)$. Relaxations induced by SNP did not differ significantly between adventitial and endothelial exposure to this agent. Release of NO from SNP requires the presence of reducing agents in biological tissues and does not occur in the dark in the absence of tissue (13). Thus, SNPinduced relaxations in the current study most likely occurred after SNP diffused across the adventitia and released NO either within or in close proximity to the smooth muscle cells. The similarity of relaxations between endothelial and adventitial delivery of SNP indicate that the adventitia does not act as a diffusion barrier to this agent, and that NO is released after the SNP has crossed the adventitia. $\mathrm{CO}$, an agent with very similar physical properties to NO, did not relax vessels differently depending upon the side from which it was administered. $\mathrm{CO}$ binds to the heme moiety of guanylate cyclase (1) and activates this enzyme for the production of cyclic GMP (17). CO reportedly causes relaxation of porcine coronary arteries (18) and inhibition of platelet aggregation (19) by activating guanylate cyclase. In marked contrast to NO, we observed that relaxations induced by $\mathrm{CO}$ were similar between endothelial and adventitial exposures to this agent. Moreover, placement of a layer of pericardium over the endothelial surface did not alter CO-induced relaxations. NO and $\mathrm{CO}$ have mol wt of 30.01 and 28.01, respectively; solubilities in water of $4.6 / 100 \mathrm{ml}$ and $2.3 / 100$ $\mathrm{ml}$ at $20^{\circ} \mathrm{C}$, respectively; and solubilities in ethanol of $26.6 / 100$ $\mathrm{ml}$ and $19.0 / 100 \mathrm{ml}$ at $20^{\circ} \mathrm{C}$, respectively (20). It is unlikely that these small differences in physical properties explain the presence of differential responses to $\mathrm{NO}$ as well as their absence to CO. Quantitatively, CO-induced relaxations were significantly smaller in magnitude than NO-induced relaxations (endothelial delivery, Figs. 4 and 7). The lower solubility of $\mathrm{CO}$ in both water and ethanol compared with NO may be partly responsible for this observation. In addition, the overall stability of NO-guanylate cyclase may be greater than $\mathrm{CO}$-guanylate cyclase. At least for hemoglobin, NO binds to the heme moiety of this molecule two orders of magnitude faster than $\mathrm{CO}$, and dissociates from hemoglobin 20 times more slowly than $\mathrm{CO}(4)$.

Other vasoactive agents also display differences between endothelial and adventitial potencies. For example, Lew et al. (21) reported that small arterioles in hamster cheek pouch are 100 times more sensitive to phenylephrine when applied to the adventitial surface than when applied to the endothelial surface. The adventitial potency was highly correlated with the octanol/ water partition coefficient of phenylephrine and several vasoactive agents. They concluded that the endothelium forms a barrier to the diffusion of small water soluble molecules from the lumen to the smooth muscle layer. Because the ethanol/ water partition coefficients of $\mathrm{NO}(6.0)$ and $\mathrm{CO}(8.3)$ are quite similar, it does not appear that this factor alone can account for the differences in adventitial versus endothelial potencies of $\mathrm{NO}$ and $\mathrm{CO}$ in the current study.

The above results indicate that the adventitia acts as a barrier specific to NO. The major difference between NO and CO is that NO is a highly reactive free radical. We hypothesize that NO reacts with and is inactivated by some component of the adventitia, thereby reducing its effective concentration, whereas CO and SNP freely pass through this layer. Potential inactivating mechanisms might include superoxide- or transition metalcontaining proteins. Although SOD was used in all experiments, the concentration might not have been sufficient to counteract adventitial superoxide production. Since the adventitia is much thicker than the single-cell endothelial layer, its barrier effect may be primarily due to its increased path length. However, reports that the single-cell airway epithelial layer also acts as a barrier to NO relaxations of tracheal smooth muscle indicate that this effect may be much more specific than simple path length (22). Although repetitive adventitial exposure to 965 ppm of NO failed to induce any additional relaxation in the current study, $10 \%$ (100,000 ppm) NO did pass through, indicating that the adventitial barrier may be saturable at very high concentrations of NO. It should also be noted that the tissue holder developed for this study is restricted to the use of vessels with inside diameters of $2 \mathrm{~mm}$ or greater. Whether vessels smaller than this also display the same diminished adventitial potency of NO will require the use of other techniques.

The differential response to NO observed in the current study provides evidence for a highly localized regulation of pulmonary vascular tone by NO synthesized by the endothelium. Such NO would not be expected to have distant effects because of two mechanisms: first, as shown in this current study, it will have difficulty crossing the adventitia to affect extravascular tissues or distant vessels; second, if it enters the vessel lumen it will be rapidly cleared by hemoglobin $(4,23)$. Conversely, NO generated by extravascular sources may have limited access to pulmonary vascular smooth muscle because of the adventitial barrier. For instance, extravascular cells such as pulmonary macrophages can be induced to produce large quantities of NO $(24,25)$, yet pulmonary vasodilation is not typically seen in diseases where macrophages are induced $(26,27)$.

It is widely presumed that NO, delivered as an inhaled gas for the treatment of newborn and adult pulmonary hypertension, simply diffuses through the pulmonary interstitium and the vascular adventitia to activate smooth muscle guanylate cyclase in 
pulmonary arterioles (10). However, no direct evidence exists for this proposed movement and reaction of NO in vivo. We propose three ways to reconcile our data with the ability of inhaled NO to cause pulmonary vasodilation. First, inhaled NO may simply overwhelm the adventitial barrier, especially of very small vessels. Concentrations of inhaled NO (5-80 ppm) used to dilate the pulmonary circulation are 1,000-fold greater than concentrations spontaneously exhaled from the lungs (8$50 \mathrm{ppb}$ ) (28). Similarly, our data indicate that 1,000 times greater concentrations of NO are required to dilate isolated pulmonary arteries when given from the adventitial side than when given from the endothelial side. Second, the actual site of the pulmonary circulation dilated by inhaled NO is not known. While it is presumed that inhaled NO has its effect directly on pulmonary arterial smooth muscle, we and others have observed pulmonary veins to be more sensitive to NO than pulmonary arteries (29-31). Perhaps inhaled NO has its effect in part on venules just downstream of the alveolar-capillary junction. Small changes in pulmonary venous pressure have been reported to produce large changes on proximal arteriolar pressure (32). While NO binds avidly to hemoglobin, it may bind less effectively to hemoglobin well saturated with oxygen as it leaves the alveolar capillaries (33). Finally, recent reports suggest that inhaled NO reacts with thiol constituents of airway lining fluid, forming high concentrations of stable, bioactive nitrosothiol compounds (34). Nitrosothiols have been demonstrated to be more potent and stable in bioassay systems than authentic NO (35). The vascular effect of inhaled NO might be accomplished by a stable bioactive intermediary, which can pass through the adventitial barrier as SNP does. Further exploration of potential mechanisms by which inhaled NO causes pulmonary vasodilation will be important to our understanding of this therapy.

\section{Acknowledgments}

The authors wish to thank Elisabeth Giese and Sylvia Gugino for their invaluable technical assistance in the design and performance of these experiments.

This study was supported in part by grants HL-28669 and HL-41387 from the National Institutes of Health.

\section{References}

1. Ignarro, L. J. 1991. Signal transduction mechanisms involving nitric oxide Biochem. Pharmacol. 41:485-490.

2. Moncada, S., R. M. J. Palmer, and E. A. Higgs. 1991. Nitric oxide: physiology, pathophysiology, and pharmacology. Pharmacol. Rev. 43:109-142.

3. Palmer, R. M. J., A. G. Ferrige, and S. A. Moncada. 1987. Nitric oxide release accounts for the biological activity of endothelium-derived relaxing factor Nature (Lond.). 327:524-526.

4. Henry, Y., M. Lepoivre, J. C. Draper, C. Ducrocq, J. L. Boucher, and A. Guissani. 1993. EPR characterization of molecular targets for NO in mammalian cells and organelles. FASEB (Fed. Am. Soc. Exp. Biol.) J. 7:1124-1134.

5. Nathan, C. 1992. Nitric oxide as a secretory product of mammalian cells. FASEB (Fed. Am. Soc. Exp. Biol.) J. 6:3051-3054.

6. Bernard, C., B. Szekely, I. Philip, E. Wollman, D. Payen, and A. Tidgui. 1992. Activated macrophages depress the contractility of rabbit carotids via an L-arginine/nitric oxide-dependent effector mechanism. J. Clin. Invest. 89:851860.

7. Bissonnette, E. Y., C. M. Hogaboam, J. L. Wallace, and A. D. Befus. 1991. Potentiation of tumor necrosis factor-alpha-mediated cytotoxicity of mast cells by their production of nitric oxide. J. Immunol. 147:3060-3065.

8. McCall, T. B., N. K. Boughton-Smith, R. M. J. Palmer, B. J. R. Whittle, and S. Moncada. 1989. Synthesis of nitric oxide from L-arginine by neutrophils Biochem. J. 261:293-296.

9. Busse, R., and A. Mulsch. 1990. Induction of nitric oxide synthase by cytokines in vascular smooth muscle. FEBS (Fed. Eur. Biochem. Soc.) Lett. 275:87-90.

10. Fratacci, M. D., C. G. Frostell, T. Y. Chen, J. C. Wain, D. R. Robinson, and W. M. Zapol. 1991. Inhaled nitric oxide: a selective pulmonary vasodilator of heparin-protamine vasoconstriction in sheep. Anesthesiology. 75:990-999.

11. Zayek, M., D. Cleveland, and F. C. Morin III. 1993. Treatment of persistent pulmonary hypertension in the newborn lamb by inhaled nitric oxide. J. Pediatr. 122:743-750.

12. Zayek, M., L. Wild, J. D. Roberts, and F. C. Morin III. 1993. Effect of nitric oxide on the survival rate and incidence of lung injury in newborn lambs with persistent pulmonary hypertension. J. Pediatr. 123:947-952.

13. Bates, J. N., M. T. Baker, R. Guerra, and D. G. Harrison. 1991. Nitric oxide generation from nitroprusside by vascular tissue. Biochem. Pharmacol. 42:S157-S165.

14. Vedernikov, Y. P., T. Graser, and N. Tiedt. 1987. Is there an abluminal release of endothelial-derived relaxing factor (EDRF)? Basic Res. Cardiol. 82:172-177.

15. Bassenge, E., R. Busse, and U. Pohl. 1987. Abluminal release and asymmetrical response of the rabbit arterial wall to endothelium-derived relaxing factor. Circ. Res. 61 (Suppl II):II-68-II-73.

16. Waldman, S. A., and F. Murad. 1987. Cyclic GMP synthesis and function. Pharmacol. Rev. 39:163-196.

17. Schmidt, H. H. H. W. 1992. Endogenous soluble guanylyl cyclase-activating factors. FEBS (Fed. Eur. Biochem. Soc.) Lett. 307:102-107.

18. Graser, T., Y. P. Vedernikov, and B. S. Li. 1990. Study on the mechanism of carbon monoxide induced endothelium-independent relaxation in porcine coronary artery and vein. Biomed. Biochim. Acta. 49:293-296.

19. Brune, B., and V. Ullrich. 1987. Inhibition of platelet aggregation by carbon monoxide is mediated by activation of guanylate cyclase. Mol. Pharmacol. 32:497-504.

20. Stephen, H., and T. Stephen. 1963. Solubilities of Organic and Inorganic Compounds. Macmillian Press, New York.

21. Lew, M. J., R. J. Rivers, and B. R. Duling. 1989. Arteriolar smooth muscle responses are modulated by an intramural diffusion barrier. Am. J. Physiol. 257:H10-H16.

22. Munakata, M., Y. Masaki, I. Sakuma, H. Ukita, Y. Otsuka, Y. Homma, and Y. Kawakami. 1990. Pharmacological differentiation of epithelium-derived relaxing factor from nitric oxide. J. Appl. Physiol. 69:665-670.

23. Wennmalm, A., G. Benthin, and A. S. Petersson. 1992. Dependence of the metabolism of nitric oxide (NO) in healthy human whole blood on the oxygenation of its red cell haemoglobin. Br. J. Pharmacol. 106:507-508.

24. Marletta, M., P. Yoon, R. Iyengar, C. Leaf, and J. Wishnok. 1988. Macrophage oxidation of L-arginine to nitrite and nitrate: nitric oxide is an intermediate. Biochemistry. 27:8706-8711.

25. Vodovotz, Y., N. Kwon, M. Pospischil, J. Manning, J. Paik, and C. Nathan. 1994. Inactivation of nitric oxide synthase after prolonged incubation of mouse macrophages with IFN-gamma and bacterial lipopolysaccharide. J. Immunol. 152:4110-4118.

26. Demling, R. 1993. Adult respiratory distress syndrome: current concepts. New Horizons. 1:388-401.

27. Zapol, W., and W. Hurford. 1993. Inhaled nitric oxide in the adult respiratory distress syndrome and other lung diseases. New Horizons. 1:638-650.

28. Persson, M. G., N. P. Wiklund, and L. E. Glustafsson. 1993. Endogenous nitric oxide in single exhalations and the change during exercise. Am. Rev. Respir. Dis. 148:1210-1214.

29. Gordon, J. B., and M. L. Tod. 1993. Effects of $N$-nitro-L-arginine on total and segmental vascular resistances in developing lamb lungs. J. Appl. Physiol. 75:76-85.

30. Nelin, L. D., and C. A. Dawson. 1993. The effect of Nitro-L-arginine methylester on hypoxic vasoconstriction in the neonatal pig lung. Pediatr. Res. 34:349-353.

31. Steinhorn, R., F. C. Morin III, S. Gugino, E. C. Giese, and J. A. Russell. 1993. Developmental differences in endothelium-dependent responses in isolated ovine pulmonary arteries and veins. Am. J. Physiol. 264:H2162-H2167.

32. LaBourene, J., J. Coles, D. Johnson, A. Mehra, F. Keeley, and M. Rabinovitch. 1990. Alterations in elastin and collagen related to the mechanism of progressive pulmonary venous obstruction in a piglet model. Circ. Res. 66:438-456.

33. Iwamoto, J., and F. C. Morin III. 1993. Nitric oxide inhibition varies with hemoglobin saturation. J. Appl. Physiol. 75:2332-2336.

34. Gaston, B., J. Reilly, J. Drazen, J. Fackler, P. Ramdev, D. Arnelle, M. Mullins, D. Sugarbaker, C. Chee, and D. Singel. 1993. Endogenous nitrogen oxides and bronchodilator S-nitrosothiols in human airways. Proc. Natl. Acad. Sci. USA. 90:10957-10961.

35. Myers, P., R. Minor, R. Guerra, J. Bates, and D. Harrison. 1990. Vasorelaxant properties of the endothelium-derived relaxing factor more closely resemble S-nitrosocysteine than nitric oxide. Nature (Lond.). 345:161-163. 